\title{
Numerical Simulation and Application Analysis on Characteristic of Coherent Jet and Traditional Supersonic Jet
}

\author{
Guocheng Lv,a, ${ }^{1,}$ Kun Liu ${ }^{2, b}$, Liming Bao ${ }^{1, c}$ \\ ${ }^{1}$ Jilin Professional Technology College of Electronic Information, Hang Yang Street,Jilin , China \\ ${ }^{2}$ Faculty of Materials and Metallurgy , Liaoning University of Science and Technology, Qian Shan \\ Street,Anshan , China) \\ a 83629348@qq.com, ${ }^{\mathrm{b}}$ liukun6512@163.com, ${ }^{\mathrm{c}} 123928825 @ q q . c o m$
}

Keywords:Coherent Jet,Supersonic Jet,Jet Characteristic,Numerical Simulation,Application Analysis

\begin{abstract}
We deeply simulated and analyzed the characteristics of the flow field on traditional supersonic jet and coherent jet through the computer numerical simulation software Fluent. The simulation and analysis were carried out mainly on the axial attenuation and radial expansion of the jet, which proved the superiority of coherent jet and provided theoretical basis for the practical application about coherent jet technology.
\end{abstract}

\section{Introduction}

Coherent jet technology is a new type of oxygen blowing technology developed by Praxair, USA, and has been patented ${ }^{[1]}$. This technique uses the principle of gas mechanics, adding a concomitant flow system around a conventional lance, which slows down the decay rate of the oxygen jet and forms a technique similar to the oxygen jet of a laser beam ${ }^{[2]}$. This jet has concentrated energy and strong penetrating ability, has a very obvious effect on promoting steel slag reaction, uniformizing steel water composition and temperature, reducing splashing, improving oxygen utilization rate, and increasing metal yield.

In this paper, we deeply simulated and analyzed the flow field characteristics of the traditional supersonic jet and the coherent jet through the computer simulation software Fluent. The simulation is mainly carried out from the axial attenuation and radial expansion of the jet, through the cloud map and etc. The simulation results of the value line diagram show that the coherent jet is slower in axial attenuation and the radial expansion is not obvious, which proved superiority of the polymerization jet and provided a theoretical basis for the practical application of the technology.

\section{Establishment of geometric models}

The geometric model of the traditional supersonic jet processes the jet after the Laval nozzle exit into a free jet, and the nozzle is connected to an infinite space to provide sufficient space for the full development of the jet. The calculated length in the axial direction of the jet is $2200 \mathrm{~mm}$ and the calculated length in the radial direction is $800 \mathrm{~mm}$ (sufficiently larger than the diameter of the Laval tube $22 \mathrm{~mm}$ ). The geometric model of the polymeric jet is a concomitant flow inlet around a conventional supersonic jet. The meshing of the geometric model of the specific jet is shown in Figure 1 and Figure 2 respectively. 


\section{Numerical Simulation on Characteristic of Coherent Jet and Traditional Supersonic Jet}

\subsection{Comparison of the attenuation of the jet in the axial direction}

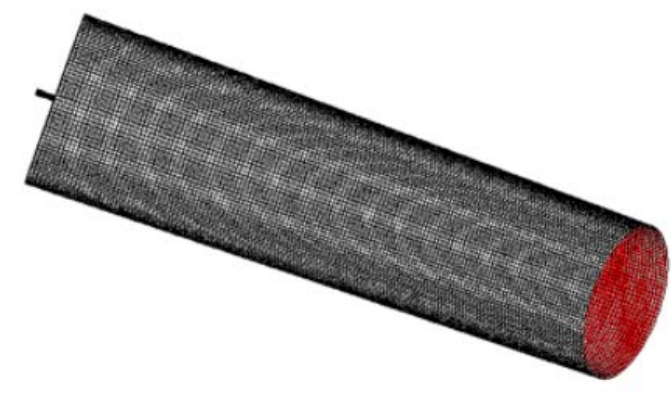

$2 \sqrt{x}$

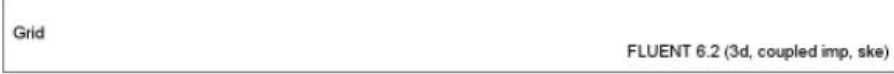

Figure 1 The grid division of the traditional oxygen lance jet model

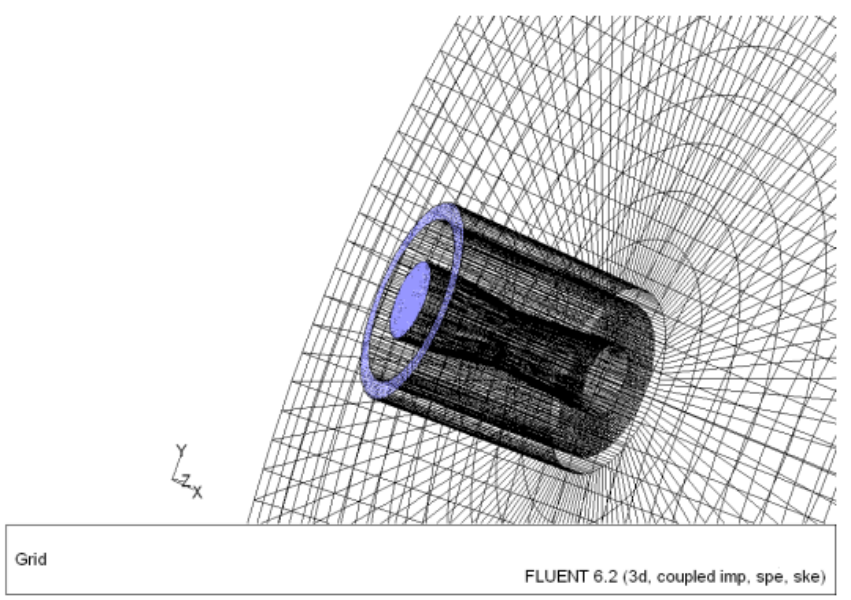

Figure 2 The grid division of coherent jet oxygen lance model

In order to better study the characteristics of the jet and highlight the superiority and application prospect of the coherent jet, this paper will compare the axial attenuation and radial expansion of the coherent jet with the traditional supersonic jet from the actual steel making conditions. To compare the two jets, first determine that the simulated process parameters are the same. Considering the actual smelting situation, the ambient temperature was chosen to be $1873 \mathrm{~K}$, the gas composition of the main orifice was oxygen, the inlet stagnation pressure was set to $0.8 \mathrm{MPa}$, and the temperature was set to $298 \mathrm{~K}$. The gas component of the sub-hole is helium gas, and the inlet stagnation pressure is set to $0.2 \mathrm{MPa}$. Considering the actual combustion temperature, the temperature of the sub-tank gas is set to $2500 \mathrm{~K}^{[3-4]}$.

The specific simulation results are shown in Figure 3 and Figure4: 


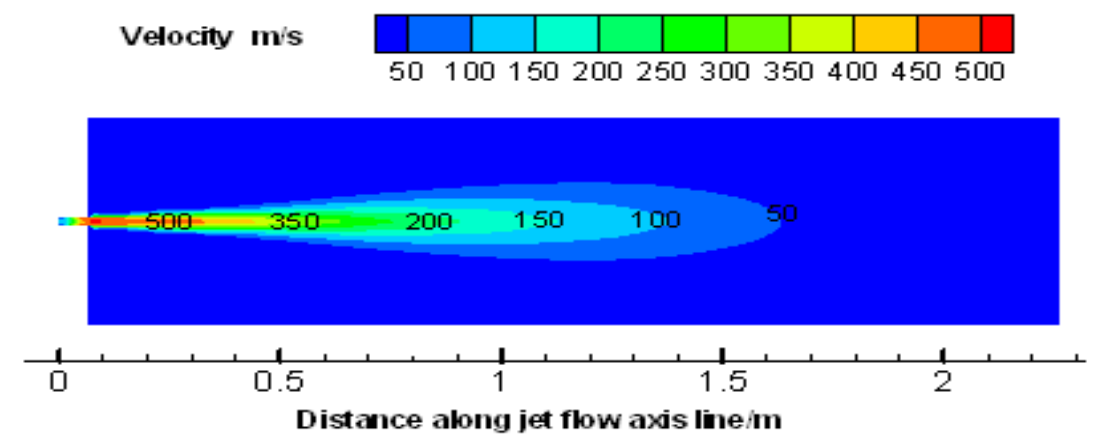

(a)supersonic jet

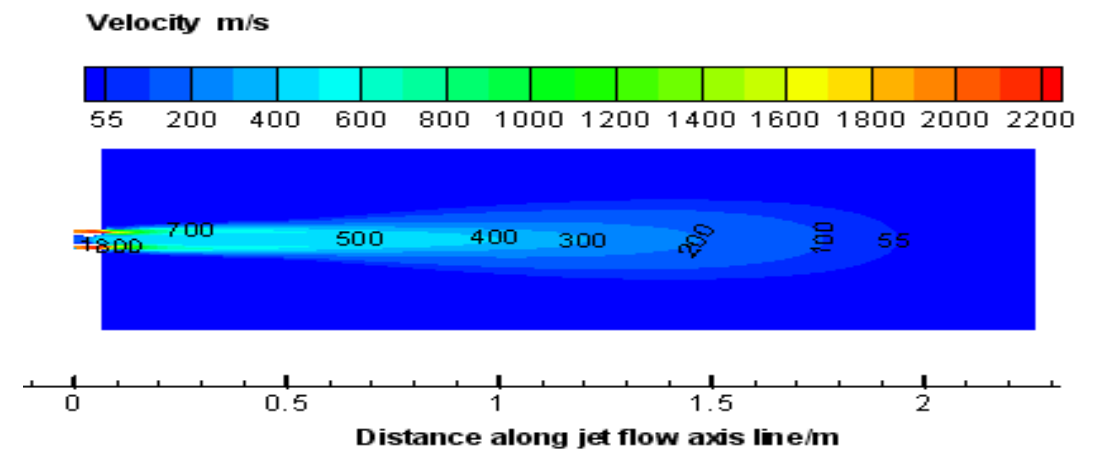

(b)coherent jet

Figure 3 Velocity cloud of jet at axial center section

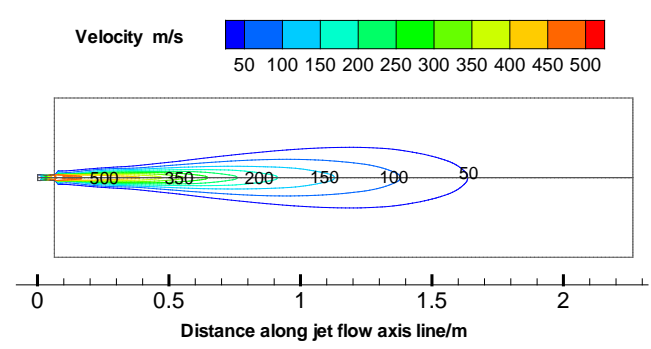

(a)supersonic jet

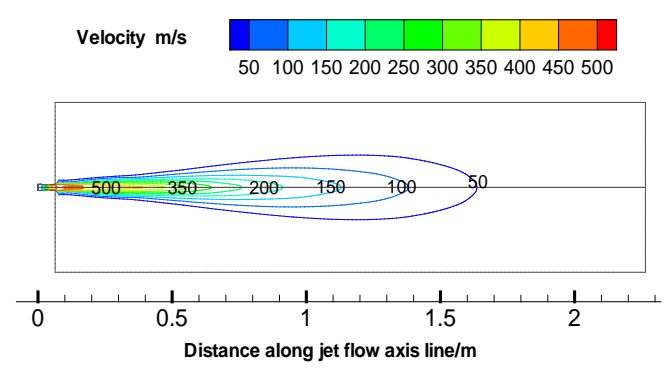

(b)coherent jet

Figure 4 Distribution magnitude of velocity at axial center section

It can be seen from Figure3 and Figure4 that the velocity of the conventional jet is reduced to 50 $\mathrm{m} \cdot \mathrm{s}^{-1}$ at $1.6 \mathrm{~m}$ on the axis, and the velocity on the axis of the coherent jet is more than $2 \mathrm{~m}$ to be attenuated to $50 \mathrm{~m} \cdot \mathrm{s}^{-1}$. The supersonic region of the coherent jet can reach more than $1 \mathrm{~m}$ in length, while the traditional jet is within $0.5 \mathrm{~m}$. This indicates that the presence of the secondary orifice accompanying the flow slows the velocity decay of the coherent jet in the axial direction. Due to the high temperature of the secondary orifice helium, the velocity can reach $2000 \mathrm{~m} \cdot \mathrm{s}^{-1}$ or more. Such a high speed has a good protection effect on the central main jet, so that the attenuation of the coherent jet is slower than that of the traditional jet. Obviously, if the two are blown at the same high gun position, the coherent jet will have a greater impact speed. 


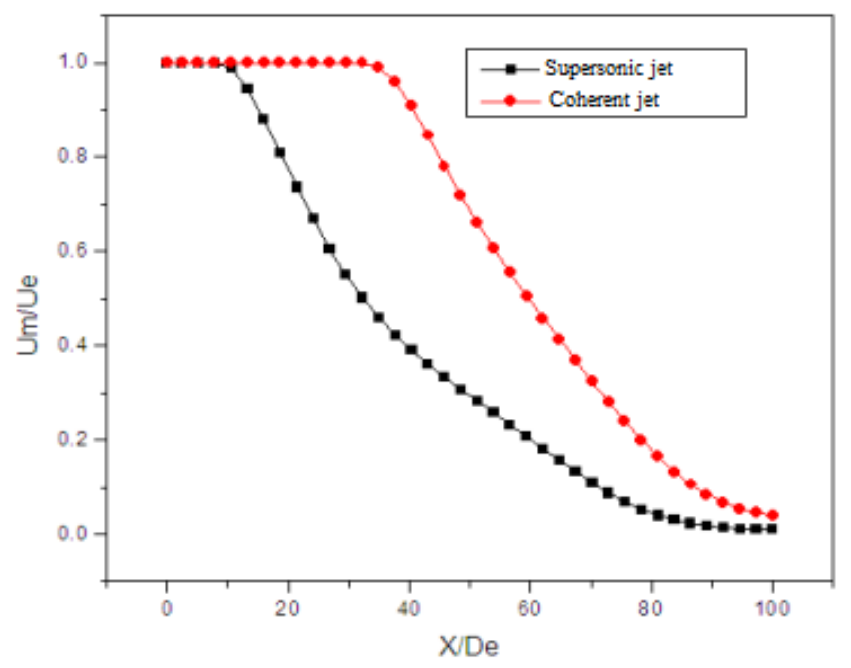

Figure 5 The comparison of velocity weakening curve between coherent jet and supersonic jet at axis section: X-distance of any point at jet axial to outlet, De- diameter of outlet of oxygen lance nozzle

By comparing the curve of the coherent jet with the velocity attenuation of the traditional jet on the axis (Figure 5), it can be seen that the jet maintains a core region at the exit, and the core region of the traditional supersonic jet has a length of about 10De, and then the velocity is rapidly attenuated. After reaching 35De, the attenuation becomes slower, and after reaching 80De, the speed is almost attenuated to zero. For the coherent jet, its core zone length is 35De, and the core zone is nearly linearly attenuated behind.

\subsection{Comparison of the expansion of the jet radial}

It can be seen from the axial attenuation of the jet that the coherent jet begins to decay after exiting the outlet 35De. Therefore, for the study of the expansion in the radial direction, the jet cross sections from the outlets 40De, 60De and 80De are selected as the research target [5]. The specific results are shown in Figure 6.
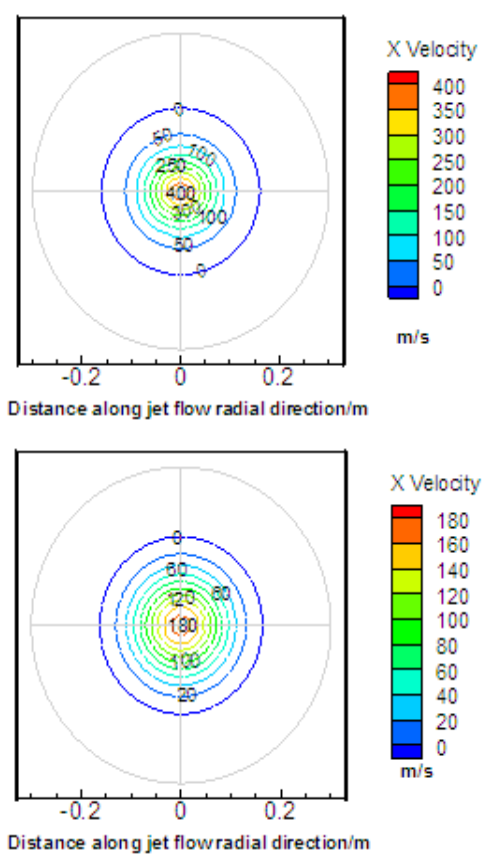

(a) $\mathrm{X}=40 \mathrm{De}$
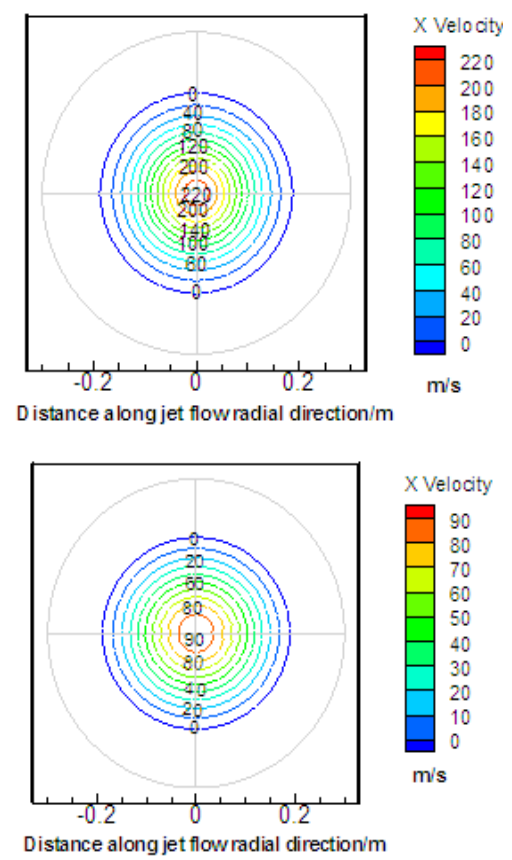

(b) $\mathrm{X}=60 \mathrm{De}$

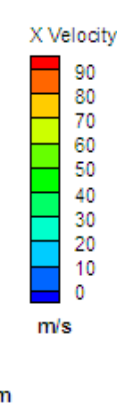

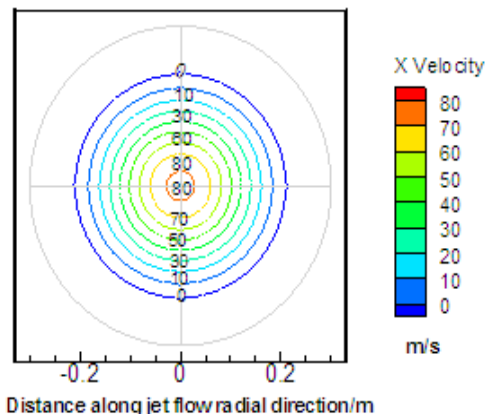

Distance along jet flow ra dial direction $/ \mathrm{m}$

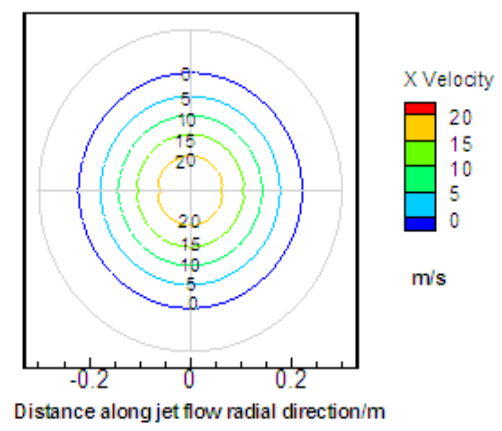

(c) $\mathrm{X}=80 \mathrm{De}$

Figure 6 Distribution magnitude of velocity at different radial center section of coherent jet and traditional supersonic jet 
It can be seen directly from Figure 6 that the affected areas of the two jets are basically the same, and the section speed is within $0.2 \mathrm{~m}$ to complete the attenuation from the center to the edge, but the center velocity of the jet in the same section is higher than the traditional one.so it decays faster. Through this phenomenon, it can be concluded that the affected area of the coherent jet is not much larger than that of the traditional jet. The jet must maintain its original diameter at a long distance from the exit. Due to the high velocity of the jet center, a jet similar to a laser beam will have a strong penetrating power to the molten pool, thus achieving a strong agitation effect on the steel making bath.

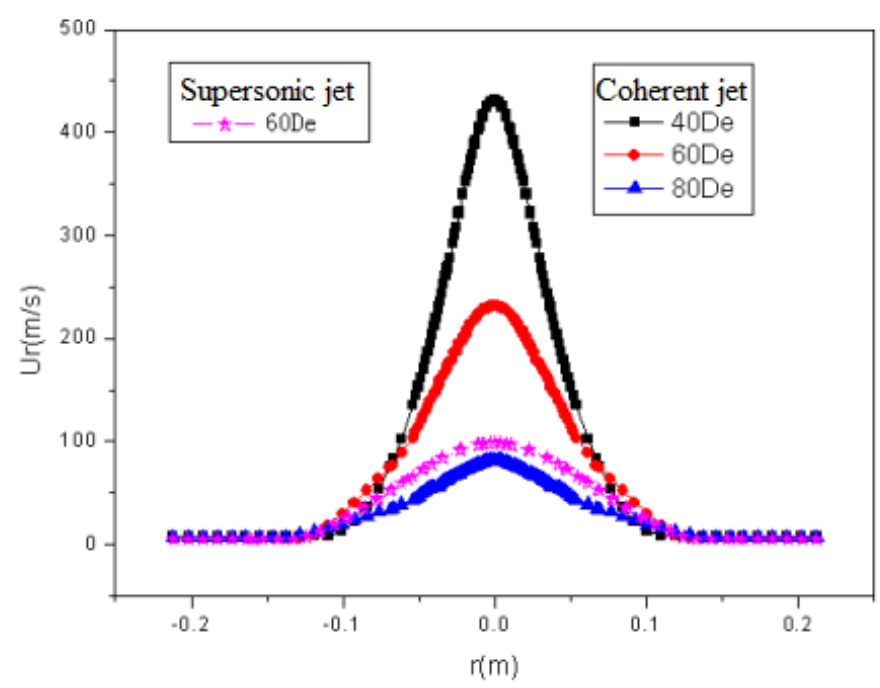

Figure 7 The comparison of velocity distribution on different radial section between coherent jet and traditional supersonic jet

From the comparison of the velocity distribution of the two jets on different radial sections (Figure 7 ), it can be concluded that the center velocity of the coherent jet at the exit 40De can reach $400 \mathrm{~m} \cdot \mathrm{s}^{-1}$ or more, still in supersonic flow, The speed at 60De is attenuated to $250 \mathrm{~m} \cdot \mathrm{s}^{-1}$. If this is positioned as the actual steel gun position, the coherent jet will still have a strong impact on the steelmaking pool and will not cause splashing. The traditional supersonic jet has been attenuated to below $100 \mathrm{~m} \cdot \mathrm{s}^{-1}$. The speed expansion of the traditional jet at 60De is similar to that of the 80D of the coherent jet.

\section{Conclusion}

Through the establishment of the coherent jet lance model, this paper mainly analyzes the axial attenuation and radial expansion of the jet, and draws the following conclusions:

(1)The axial attenuation speed of the coherent jet is slower than that of the traditional supersonic jet, and the length of the supersonic region can reach more than $1 \mathrm{~m}$. If the two are blown at the same high gun position, the coherent jet will have a greater impact velocity on the molten pool.

(2)The affected area of the coherent jet is not much larger than that of a traditional jet, and the jet maintains its original diameter for a long distance from the exit. Due to the high velocity of the jet center, a jet similar to a laser beam can be produced, which has a strong penetrating power and thus achieves a strong stirring effect on the steel making molten pool.

\section{References}

[1]ANDERSONJE, MATHURPC, SELINESR. J, Method for Introducing Gas into a Liquid [P]. U.S.Patent, No. 5814125, 1998-09-29.

[2] Changjian Cheng, Minggang Shen, Zaijing Su, Characteristics of coherent jet oxygen lance technology and its application [J]. Steel making, 2002, 18(5): 47-49. 
[3]Guocheng Lv, Fundamental study on jet characteristics of supersonic coherent jet oxygen lance [D], Anshan, Liaoning University of Science and Technology, 2009.

[4] Liming Bao, Kun Liu, Guocheng Lv, Numerical simulation of jet characteristics of supersonic oxygen lance[J], Special Steel, 2014.8. Volume 35, Issue 4: 1 -4.

[5]Kun Liu, Mathematical physics simulation of the characteristics of supersonic coherent jet oxygen gun shooting[D],Shenyang, Northeastern University, 2008 